\title{
Correction to: Enabling Drones Collaboration in ITS Applications Using a BDI Architecture Based on a 5-Dimensional Social Model
}

Hana Gharrad, Nafaa Jabeur, Ansar Ul-Haque Yasar,

Khalid Ali Sulaiyam Al Abri, Youssef El-Hansali, and Bruno Kochan

\section{Correction to: \\ Chapter "Enabling Drones Collaboration in ITS Applications Using a BDI Architecture Based on a 5-Dimensional" in: K. Arai et al. (Eds.): Proceedings of the Future Technologies Conference (FTC) 2020, Volume 1, AISC 1288, https://doi.org/10.1007/978-3-030-63128-4_5}

In the original version of the book, the Chapter 5 title was inadvertently published without the last two words, which has now been changed from "Enabling Drones Collaboration in ITS Applications Using a BDI Architecture Based on a 5Dimensional" to "Enabling Drones Collaboration in ITS Applications Using a BDI Architecture Based on a 5-Dimensional Social Model". The chapter has been updated with the changes. 